\title{
Bovisa: A Park for Work and Research
}

\author{
Domenico Chizzoniti, Luca Monica, Tomaso Monestiroli and Raffaella Neri
}

\begin{abstract}
Some projects for the "Goccia di Bovisa" (Drop) area and the neighbouring district are shown here: they fall within the urban transformation areas of the Milanese Territorial Government Plan (PGT). The Goccia area is involved in the plan to transfer part of the university campus to this site. This hypothesis was promoted and supported at the end of the 1980s by a group of researchers of the Faculty of Architecture. The idea was related to a sort of polycentric territorial city. Specifically, what they promoted was a new function for the area to set a new city centre in order to redeem its peripheral condition. The main reason for this option was the availability of dismissed industrial areas and the great rail accessibility provided for by the city plans that would have guaranteed urban and territorial relations. In agreement with the Municipality in 1990 the Faculty of Architecture developed a masterplan for the new settlement, which was blocked for decades due to pollution issues in the areas. The following projects derive from a new agreement promoted by the Municipality of Milan and Politecnico in 2016.
\end{abstract}

Keywords Urban design · Architectural composition $\cdot$ Bovisa $\cdot$ University Campus

\section{The Settlement Places}

The settlement project of the new Politecnico in Bovisa is primarily the project of a part of the city; a part that has long been destined to the outskirts, linked to the factories and their residences, emblematic of the last century's disorderly and separate growth from the city.

D. Chizzoniti $(\bowtie) \cdot$ L. Monica $\cdot$ T. Monestiroli $\cdot$ R. Neri

Architecture, Built Environment and Construction Engineering-ABC Department,

Politecnico di Milano, Milan, Italy

e-mail: domenico.chizzoniti@polimi.it

(C) The Author(s) 2020

S. Della Torre et al. (eds.), Buildings for Education, Research for Development, https://doi.org/10.1007/978-3-030-33687-5_9 
The far-sighted choice of the late 1980s to place the new Politecnico, an important institution in the city, there and link it to the public transport infrastructure system by rail should have redeemed this state, bringing regional-scale public activities, services, residences, public places and green spaces to it: a new centrality, therefore, was able to reorganise all those activities that are typical of the urban settlements around it. This is a great opportunity to redesign this part of town, to give it a new identity and a corresponding shape.

This change in purposes and activities located in Bovisa should have corresponded to a transformation in the character and the quality of the places able to represent the new role and the new identity of this part of the city: places for study, research, living, community life, leisure, entertainment, sports and so on, urban places, to represent the life of an important part of the city.

This transformation has not yet taken place for several reasons, and instead the process of decline has already begun due to the dismantling of the School of Civil Architecture, located in the Bovisa district for nearly two decades.

More importantly, Bovisa does not have a recognised architectural quality nowadays in terms of its places and buildings which correspond to the potential that the new Politecnico settlement could offer, together with other institutions (the Mario Negri Institute, for example). The study and research activities were positioned by renovating existing buildings, or by building them one by one again without a specific design, without modifying or modernising the overall urban structure, without adding the quality that a settlement of such selected activities could involve, and without defining new areas for the city.

Railway connections have been enhanced, making this area highly accessible from different parts of the entire Lombardy region and more, making the potential of this area even more obvious; but the Drop area has remained as an enclave, still isolated and closed within the railway fence.

The redesign of the large area included in the UTA of Bovisa and the possibility to design the vast area of the Drop, the original settlement site for the Politecnico, offers the opportunity to give a new definition to the places of this part of the city, which has the scientific and technological faculties of the Politecnico di Milano campus as a centre of value.

The following projects are just some of those deriving from the consultation promoted by the Municipality of Milan and Politecnico in 2016. Compared to the theme of the lower-level school, the settlement and the project for a university campus have to deal with other problems. Assuming a role of a major urban body the campus deals with the issue of building a central core in the city, a collective institution able, through its architecture, to define the identity and condition the destiny of a large urban district. The project is supposed to address the idea of the settlement and clarify its compositional principles to organise buildings and places that define it. 


\title{
2 The Waste Land. the Politecnico di Milano at the Bovisa District in the Gasometri Industrial Park Area, 2016
}

\author{
Design goup: D. Chizzoniti, L. Monica, R. Gabaglio, G. Guarisco, L. Jurina, M. \\ Bocciarelli, V. Donato, S. Recalcati, S. Riva, H. Pessoa \\ With: S. Cusatelli, P. Galbiati, O. Meregalli (Laboratorio informatico di \\ architettura), D. Orlandi Arrigoni, I. Sgaria, R. Zucco
}

The theme of the project "A park for research and work in Bovisa" is, from the earliest hypothesis of 1974, the theme of the projects and studies that have taken place over the years and that have made the area of gasometers an unresolved urban area. The area is still waiting for the definition of balances between the need for redemption in terms of architecture and landscape, compared to the potential opportunities for qualified training, research and economic development. The creation of a new Politecnico headquarters in the Bovisa area is an important opportunity to help overcome a crisis in the city, a crisis that threatens to alter its physiology. The hypothesis adopted is that of an industrial settlement, articulated over time as a place of production, of transformation and of exchange: a place that maintains these propulsive characteristics, transferring them to the sectors of production and culture. Starting from the awareness of the Politecnico history, which since 1974 sees the area of Bovisa as a place for research and education, the project tackles with the complexity of the intervention by reorganising it according to some strongly integrated functions. There are some basic aspects to be taken into consideration:

(a) the need for the consolidation and expansion of the Politecnico in the area of gasometers, with the integration of the Science Park;

(b) the opportunity to build a large urban park, equipped and connected to the campus rediscovered in the "waste land" of the gasometer area.

(c) Closely connected to these, the other functional nodes are achieved:

(d) the recovery, restoration and reuse of the abandoned heritage of the industrial complex of gas production, which continues today as a well-structured fragment of a city-factory;

(e) the integration between the future enlargement and that already implemented (Politecnico Lambruschini-La Masa). This structure requires consolidation, with interventions on urban public spaces for greater pedestrian accessibility and facilities for the campus life;

(f) the organisation of a linear residential park to complete the campus, with integrated services, facilities for accessibility with a new architectural structure of the FNM Bovisa railway station and the new shopping area.

(g) the renewal of accessibility and public transport for full compatibility between urban scale and new functions, with consequent decongestion of local traffic. This hypothesis is based on checking the sustainability on the urban scale of the amount of traffic generated by the new functions, by means of model simulations able to verify the local aspects and those of the areas surrounding (Figs. 1, 2, 3, $4,5)$. 


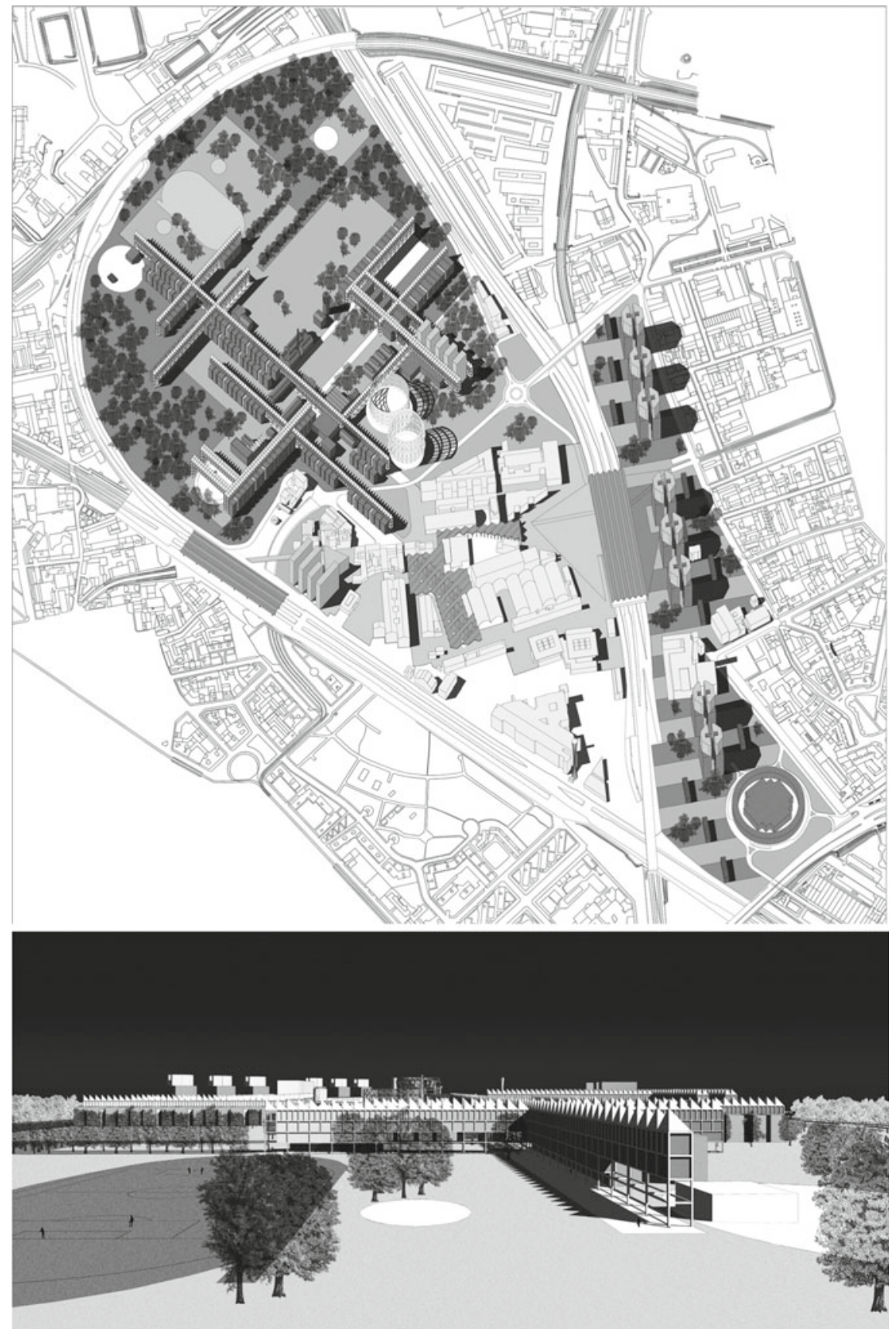

Figs. 1, 2 Axonometric view of the 'Goccia' and Perspective view 

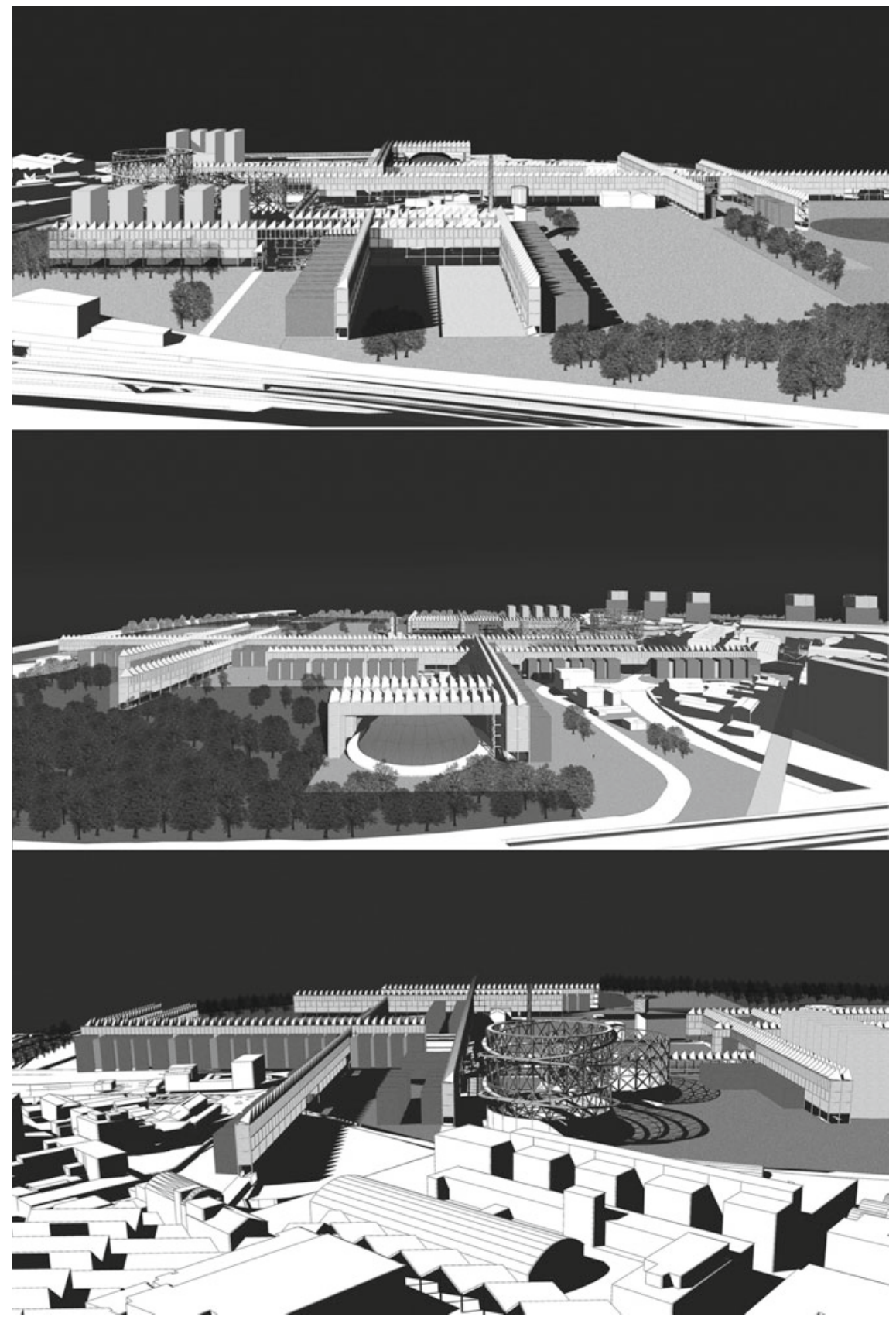

Figs. 3, 4, 5 Perspective views 


\title{
3 Bovisa 2016: A New Urban Settlement
}

\author{
Design group: R. Neri, T. Monestiroli, I. Boniello, F. Menegatti \\ With: O. Meregalli (Laboratorio LidAr), F. Guzzetti, M. Ronconi (topogra- \\ phy), Isabella Balestreri (architectural history), S. Croce, T. Poli (environmental \\ sustainability), M. Morena, T. Truppi (economic sustainability), E. Garavaglia \\ (structures), C. Campanella (restoration). Technical advisors: S. Recalacati, V. \\ Donato, S. Riva (city planning), and: G. Uboldi, E. Cuogo
}

We believe that the Drop area should not remain closed off, to be allocated exclusively for the university campus, but it should become an integral part of the city, open to life and to the inhabitants of Bovisa; it should promote a mix of activities and include collective places, open spaces and green places, measured empty spaces, corresponding to the squares and gardens of the ancient city. These open spaces, the commercial places of the city par excellence, are the elements that structure the settlement according to a hierarchical principle that distinguishes its role, character, shape and size.

The central point, organising the system, is the large rectangular lawn developed along the south-east/north-west axis, which is a recurrent orientation in Milan, where the road follows through to the previous location of the gasometers. It is concluded to the north by the library building and to the south by two gasometers, the remains and symbol of Bovisa's recent past. Inside are some of the site's most interesting industrial archaeology buildings.

All the parts that make up the new settlement converge on this site: on this side and beyond the lawn, the buildings for study and research belonging to the new Politecnico and its residences face one another.

A square with the services, commercial activities and other community spaces for the university and the neighbourhood intersects across the park and marks the central place. From the Villapizzone station, the square goes along a porticoed services building, including other buildings recovered from the former settlement and ends in another place, a triangular square with the large auditorium for the campus and the city, which the science park and business start-up accelerator buildings look out onto.

Beyond the Bovisa station, in relation to the existing district, lies a new mixed settlement that includes residences, workspaces, small shops and services. It is built along an inner central pedestrian spine, and almost parallel to Via Bovisasca, a green communal area which houses and workplaces look out onto. 
This sinuous place ends in a square which the buildings of large retailers in the vicinity of Piazzale Lugano look out onto, whose road system must be properly reorganised on the basis of the definition of the shopping centre (Figs. 6, 7, 8, 9).

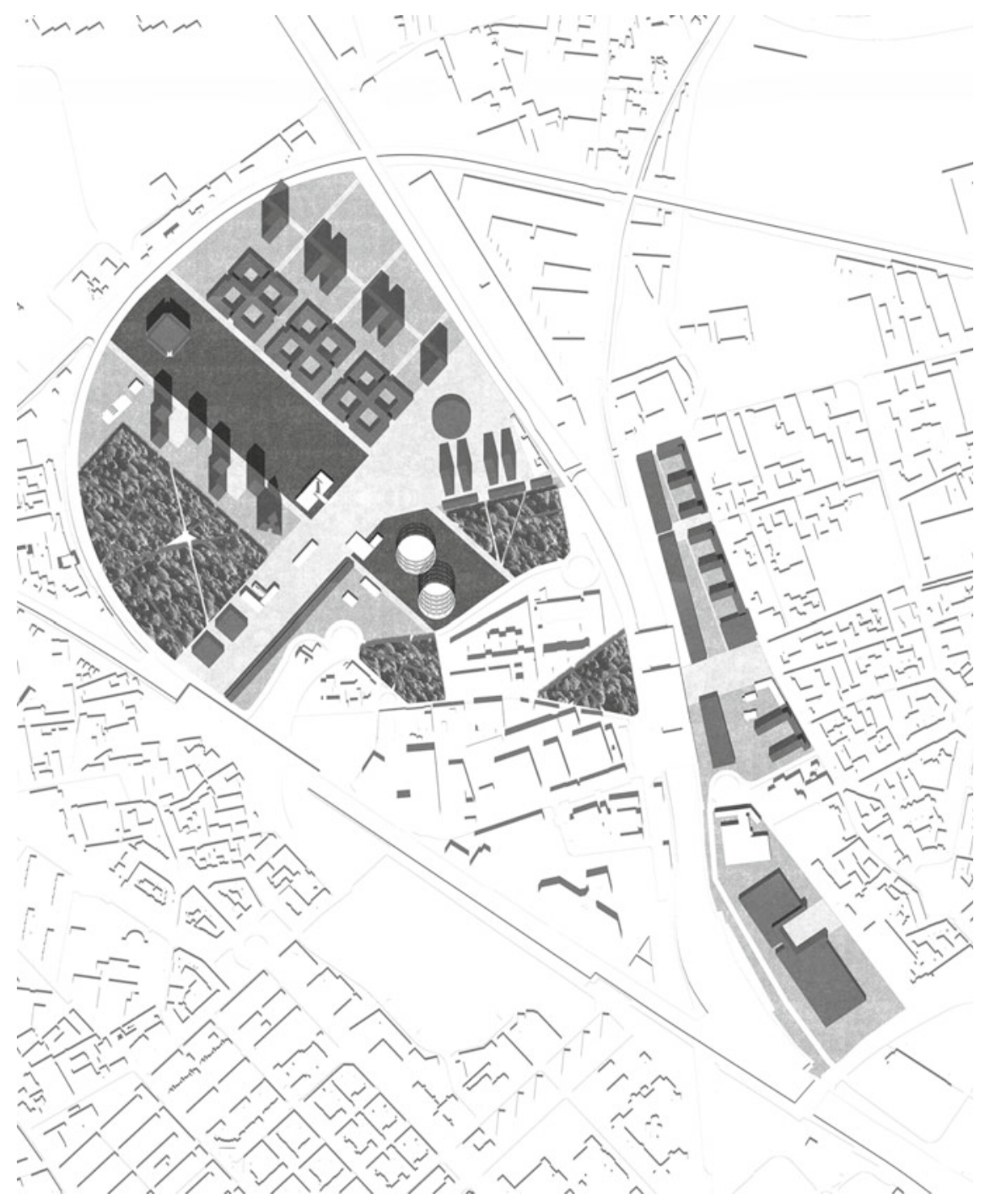

Fig. 6 General plan 


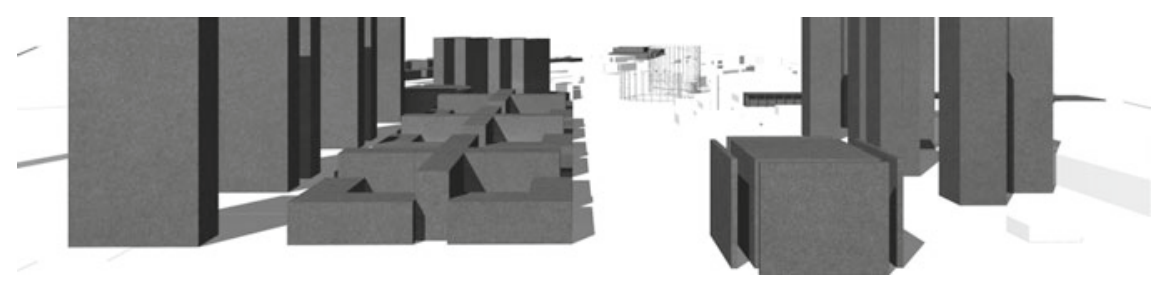

Fig. 7 Perspective view from the central lawn towards the Gasometri

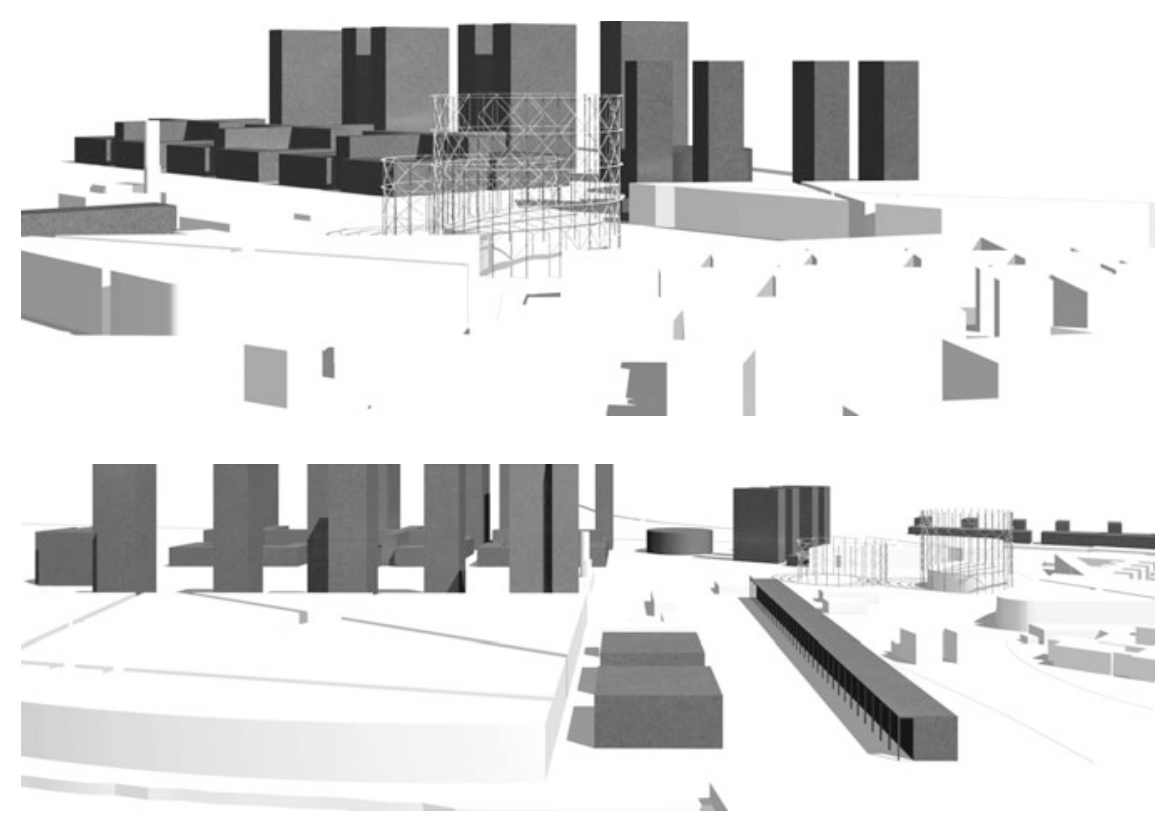

Figs. 8, 9 Perspective views from the Gasometri and from the collective plaza 
Open Access This chapter is licensed under the terms of the Creative Commons Attribution 4.0 International License (http://creativecommons.org/licenses/by/4.0/), which permits use, sharing, adaptation, distribution and reproduction in any medium or format, as long as you give appropriate credit to the original author(s) and the source, provide a link to the Creative Commons license and indicate if changes were made.

The images or other third party material in this chapter are included in the chapter's Creative Commons license, unless indicated otherwise in a credit line to the material. If material is not included in the chapter's Creative Commons license and your intended use is not permitted by statutory regulation or exceeds the permitted use, you will need to obtain permission directly from the copyright holder.

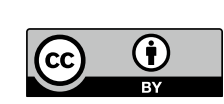

